Wilfrid Laurier University

Scholars Commons @ Laurier

1977

\title{
Probing the Phase-Dependent Conductance and Nonequilibrium Properties of Josephson Junctions by Means of Flux Entry into Weakly Closed Loops
}

James A. Blackburn

Wilfrid Laurier University, jabjabjab@cogeco.ca

H.J.T. Smith

University of Western Ontario

V. Keith

University of Western Ontario

Follow this and additional works at: https://scholars.wlu.ca/phys_faculty

\section{Recommended Citation}

Blackburn, James A.; Smith, H.J.T.; and Keith, V., "Probing the Phase-Dependent Conductance and Nonequilibrium Properties of Josephson Junctions by Means of Flux Entry into Weakly Closed Loops" (1977). Physics and Computer Science Faculty Publications. 46.

https://scholars.wlu.ca/phys_faculty/46

This Article is brought to you for free and open access by the Physics and Computer Science at Scholars Commons @ Laurier. It has been accepted for inclusion in Physics and Computer Science Faculty Publications by an authorized administrator of Scholars Commons @ Laurier. For more information, please contact scholarscommons@wlu.ca. 


\title{
Probing the phase-dependent conductance and nonequilibrium properties of Josephson junctions by means of flux entry into weakly closed loops*
}

\author{
James A. Blackburn \\ Wilfrid Laurier University, Department of Physics, Waterloo, Ontario, Canada \\ H. J. T. Smith and V. Keith \\ University of Waterloo, Department of Physics, Waterloo, Ontario, Canada
}

(Received 12 November 1976)

\begin{abstract}
Numerical simulations have shown that the relative number of flux quanta which penetrate a weakly closed superconducting loop depends upon the magnitude and sign of the phase-dependent conductance. We examine the possible use of this dependence as an experimental probe of the $\cos \varphi$ term and nonequilibrium properties of Josephson junctions.
\end{abstract}

\section{INTRODUCTION}

When an external magnetic field is applied to a multiply connected superconductor, screening currents flow so as to maintain an integral number of flux quanta within the "holes." Fluxoid quantization operates differently in weakly closed loops as was discussed by Silver and Zimmerman. ${ }^{1}$ When a superconducting ring is inter rupted by a Josephson device (point contact, microbridge, or thin-film junction), the magnetic flux inside the ring can be either a continuous or a discontinuous function of the applied field. The exact behavior depends on $L i_{c} / \phi_{0}$, where $L$ is the loop inductance, $i_{c}$ is the weak-link critical current, and $\phi_{0}$ is the flux quantum $\left(2.07 \times 10^{-15} \mathrm{~Wb}\right)$.

Previously ${ }^{2}$ we studied the dynamics of flux entry into loops which satisfied $L i_{c} \gg \phi_{0}$ and for which the junction capacitance $C$ was large. Under this latter condition the damping is light and flux enters the ring in bundles.

The total current in a Josephson junction is given by $^{3}$

$$
i=i_{c} \sin \varphi+\sigma_{1} V \cos \varphi+\sigma_{0} V,
$$

where $\varphi$ is the order-parameter phase difference, $V$ is the instantaneous voltage appearing across the junction, $\sigma_{0}$ is the normal-state junction conductance, and $\sigma_{1} \cos \varphi$ is the phase-dependent conductance. In our previous calculation the $\cos \varphi$ term was not included. However that calculation showed that the oscillating voltage could be relatively large and it is under just this condition that the $\cos \varphi$ term is expected to play an important role. Inclusion of this factor modifies the differential equation which governs the flux dynamics as follows:

$\frac{d^{2} \Phi}{d t^{2}}+\beta\left[1+\left(\frac{\sigma_{1}}{\sigma_{0}}\right) \cos (2 \pi \Phi)\right] \frac{d \Phi}{d t}+\Phi+\gamma \sin (2 \pi \Phi)=\Phi_{x}$, where $\Phi$ is the flux in the ring measured in units of $\phi_{0}, \gamma \equiv L i_{c} / \phi_{0}, \beta \equiv R^{-1}(L / C)^{1 / 2}$ is the damping coefficient, $\Phi_{x}$ is the normalized applied flux, and time is measured in units of $(L C)^{1 / 2}$.

Gayley and Wang ${ }^{4}$ have numerically solved this equation and showed that at fixed values of $L, R$, $C$, and $i_{c}$ the number of flux quanta which enter the loop $\Phi_{\text {enter }}$ is significantly different for the sample choices $\sigma_{1} / \sigma_{0}=-1,0,+1$. They also pointed out that the magnitude and sign of this ratio is still in some dispute and proposed this type of experiment as a probe of the $\cos \varphi$ amplitude. We have considered this suggestion and report here a comparison of the theory with experimental data.

\section{DISCUSSION}

From computer solutions of Eq. (2) we have generated the universal curves of $F$ ig. 1 in which $\Phi_{\text {enter }} / \gamma$ is plotted vs $\beta / \beta_{c}$. The critical damping parameter $\beta_{c}$ is defined as the maximum value of $\beta$ for which multiple quantum transitions will occur. From numerical results obtained in the range $10^{2}<\gamma<10^{5}$ we have deduced empirically that all $\Phi_{\text {enter }} / \gamma$-vs- $\beta$ curves scale as $(\gamma)^{1 / 2}$. In Fig. 2 we have plotted the dependence of $(\gamma)^{1 / 2} / \beta_{c}$ on $\sigma_{1} / \sigma_{0}$. The five points derived from simulation studies at $\gamma=1000$ are well represented by the empirical linear relationship $(\gamma)^{1 / 2} / \beta_{c}=0.20\left(\sigma_{1} / \sigma_{0}\right)+0.33$.

Measurements of $\Phi_{\text {enter }} / \gamma$ were made for both tin and lead thin-film Josephson tunnel junctions using previously reported experimental techniques. ${ }^{2}$ For these experimental data the normalized damping factor $\beta / \beta_{c}$ is determined from the expression $\beta / \beta_{c}=\left[(\gamma)^{1 / 2} / \beta_{c}\right] R^{-1}\left(\phi_{0} / C i_{c}\right)^{1 / 2}$, where $(\gamma)^{1 / 2} / \beta_{c}$ depends on the choice for $\sigma_{1} / \sigma_{0}$ as indicated in Fig. 2. The normalized experimental values of $\beta / \beta_{c}$ are plotted in Fig. 1. The choice $\sigma_{1} / \sigma_{0}=+1$ seems to yield closest agreement between experiment and 


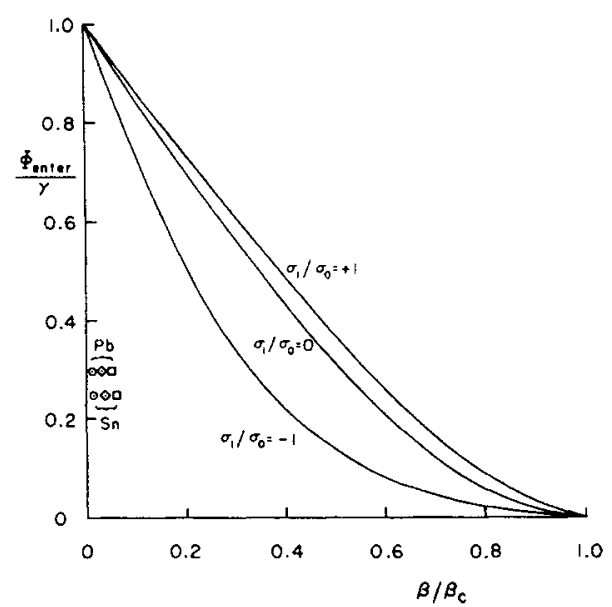

FIG. 1. Plot of the normalized number of flux quanta $\Phi_{\text {enter }} / \gamma$, which enter a superconducting loop broken by a weak link as a function of the relative damping $\beta / \beta_{c}$ for three values of the conductance ratio $\sigma_{1} / \sigma_{0}$. These curves were derived from numerical solutions of Eq. (2) in the text. Experimental points obtained from a superconducting loop interrupted by a tin or lead Josephson junction are also plotted. For either case three points are shown, corresponding to the normalizing appropriate to the possible choices $\sigma_{1} / \sigma_{0}=-1(\odot), 0(\odot),+1(\nabla)$.

theory but even this extreme value of $\sigma_{1} / \sigma_{0}$ does not produce a really satisfactory fit. The "observed" value of $\beta / \beta_{c}$ is consistently much smaller than the theoretically expected value appropriate to the measured $\Phi$ enter $/ \gamma$.

The question then arises as to the origin of such quantitative discrepancies. Let us now consider the various factors appearing in the expression for $\beta / \beta_{c}$.

There is no problem with $i_{c}$ because it is measured directly. On the other hand, $C$ is calculated using the parallel plate formula with dielectric constant $\epsilon=4$, area $A=1.7 \times 10^{-8} \mathrm{~m}^{2}$, and spacing $d=25 \times 10^{-10} \mathrm{~m}$. This yields $C \cong 2.4 \times 10^{-10} \mathrm{~F}$. It is unlikely that the uncertainty exceeds $\pm 100 \%$. Whether this parallel plate formula is applicable at the high frequencies $\left(\sim 10^{10} \mathrm{~Hz}\right)$ associated with flux entry is a problem that has been analyzed by Bassaviah and Greiner ${ }^{5}$ who examined the self-resonant peaks in a Josephson junction. They concluded that the formula was indeed correct in this type of situation. Finally the resistance $R$ must be considered. Here there is little justification for

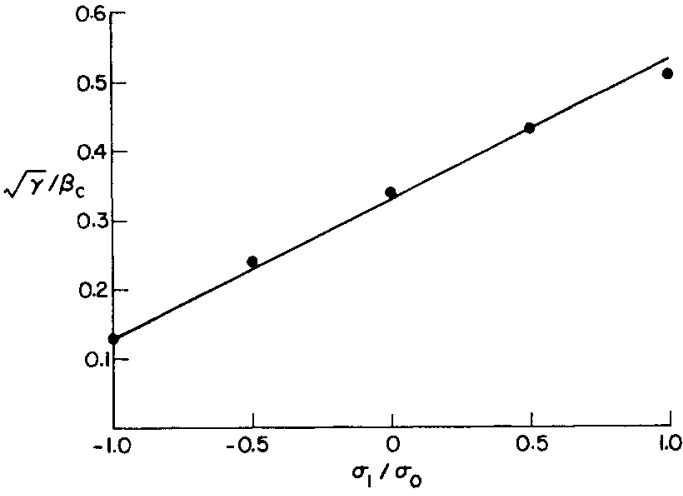

FIG. 2. Plot of $(\gamma)^{1 / 2} / \beta_{c}$ vs the conductance ratio $\sigma_{1} / \sigma_{0}$. The points are obtained from computer simulations; the straight line is a least-squares fit.

using the dc junction resistance since the flux flow mechanism leads to very high-frequency normal currents. To achieve agreement between our experiment and theory we would need to decrease $R$ by a factor of approximately 10 .

We note in this connection the recent papers by Højgard Jensen and Lindelof ${ }^{6}$ and by Clark and Lindelof ${ }^{7}$ which treat the effect of the finite relaxation time for normal electron excitations on the current voltage characteristic of resistively shunted Josephson junctions. It was concluded that the effective shunt resistance in the equivalent circuit for a microbridge could be treated as

$$
1 / R=1 / R_{N}\left(1+\frac{1}{15} \Gamma\right),
$$

where $R_{N}$ is the normal-state resistance. The dimensionless parameter $\Gamma$ was estimated for their samples in the range 50-100.

For our thin-film junctions agreement between the experimental points and the $\sigma_{1}=0$ curve in Fig. 1 can be achieved with such an equivalent resistance and $\Gamma=115$.

These experiments are more sensitive to the effects of finite lifetime than to the magnitude of the phase-dependent conductance. If $\sigma_{1} / \sigma_{0}$ can be independently determined then it will be possible to experimentally probe the nonequilibrium properties by means of the flux-entry process.

\section{ACKNOWLEDGMENT}

We thank the Computer Centre, Wilfrid Laurier University, for a grant of computer time.
*Supported by the National Research Council of Canada. ${ }^{1}$ A. H. Silver and J. E. Zimmerman, Phys. Rev. 157, 317 (1967).
${ }^{2}$ H. J. T. Smith and J. A. Blackburn, Phys. Rev. B $\underline{12}$, 940 (1975).

${ }^{3}$ B. D. Josephson, Rev. Mod. Phys. 36, 216 (1964); Adv. 
Phys. 14, 419 (1965).

${ }^{4}$ R. I. Gayley and T. C. Wang (private communication). ${ }^{5}$ S. Bassaviah and J. H. Greiner, J. Appl. Phys. $\underline{47}$, 4201 (1976).
${ }^{6} \mathrm{H}$. Hojgard Jensen and P. E. Lindelof, J. Low Temp. Phys. 23, 469 (1976).

${ }^{7}$ T. D. Clark and P. E. Lindelof, Phys. Rev. Lett. 37 , 368 (1976). 